\title{
Dilation or biodegradable stent placement for recurrent benign esophageal strictures: a randomized controlled trial
}

Authors

Daisy Walter ${ }^{1}$, Maarten W. van den Berg ${ }^{2,3}$, Meike M. Hirdes ${ }^{4}$, Frank P. Vleggaar ${ }^{1}$, Alessandro Repici ${ }^{5,6}$, Pierre H. Deprez ${ }^{7}$, Bartolomé L. Viedma ${ }^{8}$, Laurence B. Lovat ${ }^{9}$, Bas L. Weusten ${ }^{4}$, Raf Bisschops ${ }^{10}$, Rehan Haidry ${ }^{11}$, Elisa Ferrara ${ }^{5}$, Keith J. Sanborn ${ }^{12}$, Erin E. O'Leary ${ }^{12}$, Jeanin E. van Hooft ${ }^{2}$, Peter D. Siersema ${ }^{1,13}$

Institutions

1 Department of Gastroenterology and Hepatology, University Medical Center Utrecht, Utrecht, The Netherlands

2 Department of Gastroenterology and Hepatology, Amsterdam Medical Center, Amsterdam, The Netherlands

3 Department of Gastroenterology and Hepatology, HAGA Hospital, den Haag, The Netherlands

4 Department of Gastroenterology, St. Antonius Hospital, Nieuwegein, The Netherlands

5 Department of Gastroenterology, Humanitas Research Hospital, Milano, Italy

6 Department of Biomedical Science, Humanitas University, Milano, Italy

7 Department of Gastroenterology and Hepatology, Cliniques universitaires Saint-Luc, Université Catholique de Louvain, Bruxelles, Belgium

8 Department of Gastroenterology and Hepatology, Hospital General Universitario de Ciudad Real, Ciudad Real, Spain

9 Division of Surgery and Interventional Science, University College London Hospital, London, United Kingdom

10 Department of Gastroenterology, University Hospitals Leuven, KU Leuven, Leuven, Belgium

11 Department of Gastroenterology, University College London Hospital, London, United Kingdom

12 Cook Research Incorporated, West Lafayette, Indiana, United States

13 Department of Gastroenterology and Hepatology, Radboud University Medical Center, Nijmegen, The Netherlands

submitted 20.10.2017

accepted after revision $\quad 14.3 .2018$

Bibliography

DOI https://doi.org/10.1055/a-0602-4169

Published online: 2018 | Endoscopy

(c) Georg Thieme Verlag KG Stuttgart · New York

ISSN 0013-726X
Corresponding author

Peter D. Siersema, MD, Department of Gastroenterology and Hepatology, Radboud University Medical Center, Geert Grooteplein Zuid 10, 6525 GA Nijmegen, The Netherlands peter.siersema@radboudumc.nl

$\circledast$ Supplementary Table e1

Online content viewable at:

https://doi.org/10.1055/a-0602-4169

\section{ABSTRACT}

Background Dilation is the standard of care for recurrent benign esophageal strictures (BES). Biodegradable stents may prolong the effect of dilation and reduce recurrences. Efficacy and safety of dilation and biodegradable stent placement early in the treatment algorithm of recurrent BES were compared.

Methods This multicenter, randomized study enrolled patients with BES treated with previous dilations to $\geq 16 \mathrm{~mm}$. The primary end point was number of repeat endoscopic dilations for recurrent stricture within 3 and 6 months. Secondary outcomes through 12 months included safety, time to first dilation for recurrent stricture, dysphagia, and level of activity.

Results At 3 months, the biodegradable stent group ( $n=$ 32) underwent significantly fewer endoscopic dilations for recurrent stricture compared with the dilation group $(\mathrm{n}=$ 34; $P<0.001$ ). By 6 months, the groups were similar. The number of patients experiencing adverse events was similar between the groups. Two patients in the biodegradable stent group died after developing tracheoesophageal fistulas at 95 and 96 days post-placement; no deaths were attributed to the stent. Median time to first dilation of recurrent stricture for the biodegradable stent group was significantly longer (106 vs. 41.5 days; $P=0.003$ ). Dysphagia scores improved for both groups. Patients in the biodegradable stent group had a significantly higher level of activity through 12 months $(P<0.001)$. 
Conclusion Biodegradable stent placement is associated with temporary reduction in number of repeat dilations and prolonged time to recurrent dysphagia compared with dilation. Additional studies are needed to better define the exact role of biodegradable stent placement to treat recurrent BES.
Clinical.Trials.gov

NCT01337206

TRIAL REGISTRATION: This study is a multicenter, random-

ized trial.

NCT01337206 at clinicaltrials.gov

\section{Introduction}

Benign esophageal strictures (BES) occur following peptic, corrosive or radiation injury, surgical anastomosis, postmucosal resection, or esophageal inflammatory disease [1 - 3]. Dysphagia is a frequent symptom for these patients, resulting in an inability to eat a normal diet, and leading to malnutrition, weight loss, aspiration, and impaired quality of life [4, 5].

The primary treatment for BES is endoscopic dilation with balloon or bougie dilators. Although dilation relieves dysphagia in the majority of patients with BES, repeated sessions, which are a burden to patients and increase healthcare costs $[5,6]$, are frequently required [7-9]. Temporary stent placement, which dilates the stricture for a prolonged period of time and may lead to a reduction in stricture recurrence $[10,11]$, is a potential treatment for patients with strictures that are refractory to ongoing dilation. Partially and fully covered self-expandable stents require additional endoscopic procedures for removal and are prone to tissue ingrowth or migration [11-14].

To address these problems, biodegradable stents have been designed as a promising alternative. To reduce the risk of migration, the biodegradable stent has flared ends and is uncovered, allowing for tissue ingrowth. Stent integrity and radial force are typically maintained for up to 8 weeks, and considerable stent degradation is expected approximately 12 weeks following placement [15-18]. A recent study reported a median time to complete stent degradation of 127 days (range 98 219 days) [19]. Because the biodegradable stent degrades, removal is not required. Experience with biodegradable stents is limited to small case series of patients with refractory strictures [15-20]. No studies have evaluated whether biodegradable stents placed earlier in the treatment algorithm could be an effective alternative to reduce the risk of recurrent dysphagia. This study compared the efficacy and safety of standard dilation and biodegradable stent placement in patients with recurrent $\mathrm{BES}$.

\section{Methods}

\section{Study design}

Between 2012 and 2015, a multicenter, randomized controlled trial compared dilation therapy with biodegradable esophageal stent placement in patients with BES. Patients with confirmed recurrent $B E S$, a dysphagia score $\geq 2$ on the Ogilvie scale [21] and $\leq 21$ on the Dakkak and Bennett scale [22] (see - Supplementary Table e1, available online), and a history of one to five previous endoscopic dilations to $\geq 16 \mathrm{~mm}$ within the pre- vious year were eligible. Key exclusion criteria included a surgical or interventional procedure in the esophagus 30 days prior to or after the procedure; previous esophageal stent placement or dilation method other than standard bougie or balloon; stricture within $1.5 \mathrm{~cm}$ of the upper esophageal sphincter; lesions requiring more than one stent; stricture length $\geq 10 \mathrm{~cm}$; active esophageal perforation, leak, fistula, or varices; highly suspected esophageal malignancy; and known eosinophilic esophagitis or motility disorder.

Approval for the study was obtained from each site's ethics committee, and patients provided written informed consent. Permuted block randomization, using a centralized computer system, randomized patients in a 1:1 ratio to standard dilation therapy or biodegradable stent placement. The study was not blinded.

\section{Dilation and stent placement procedures}

At the physician's discretion, patients were placed under sedation prior to endoscopic procedures. A balloon or bougie was used for dilation according to standard institutional practice to reach a target diameter of $\geq 16 \mathrm{~mm}$. Stepwise dilation was permitted at the physician's discretion when a single session was considered unsafe. The target diameter had to be reached within 2 weeks. Endoscopy confirmed dilation efficacy and assessed for potential perforation.

In the stent group, pre-dilation was allowed prior to the endoscopic placement of a biodegradable stent (SX-ELLA; EllaCS, Hradec Králové, (zech Republic), which was made from polydioxanone, a biodegradable synthetic polymer. Based on initial stricture assessment, a stent of appropriate length (60, 80 , or $100 \mathrm{~mm}$ ) and diameter $(18,20$, or $23 \mathrm{~mm})$, was placed under fluoroscopic guidance. Endoscopy confirmed correct stent positioning by visualizing the radiopaque markers and expansion across the stricture ( $\mathbf{F i g . 1}$ ). Patients in both groups used a proton pump inhibitor according to standard of care.

\section{Patient follow-up}

Patients were contacted by telephone 14 days, monthly through 6 months, and at 12 months after treatment. At 3 months, patients in the stent group underwent a radiographic evaluation of the esophagus to visualize the gold markers. For those patients with visible gold markers at 3 months, radiography was performed again at 6 months. With the exception of this radiographic evaluation in patients with a biodegradable stent, the follow-up schedule was comparable between groups. Reintervention for recurrent significant dysphagia, defined as a dysphagia score $\geq 2$ on the Ogilvie scale [21] or $\leq 21$ on the Dak- 


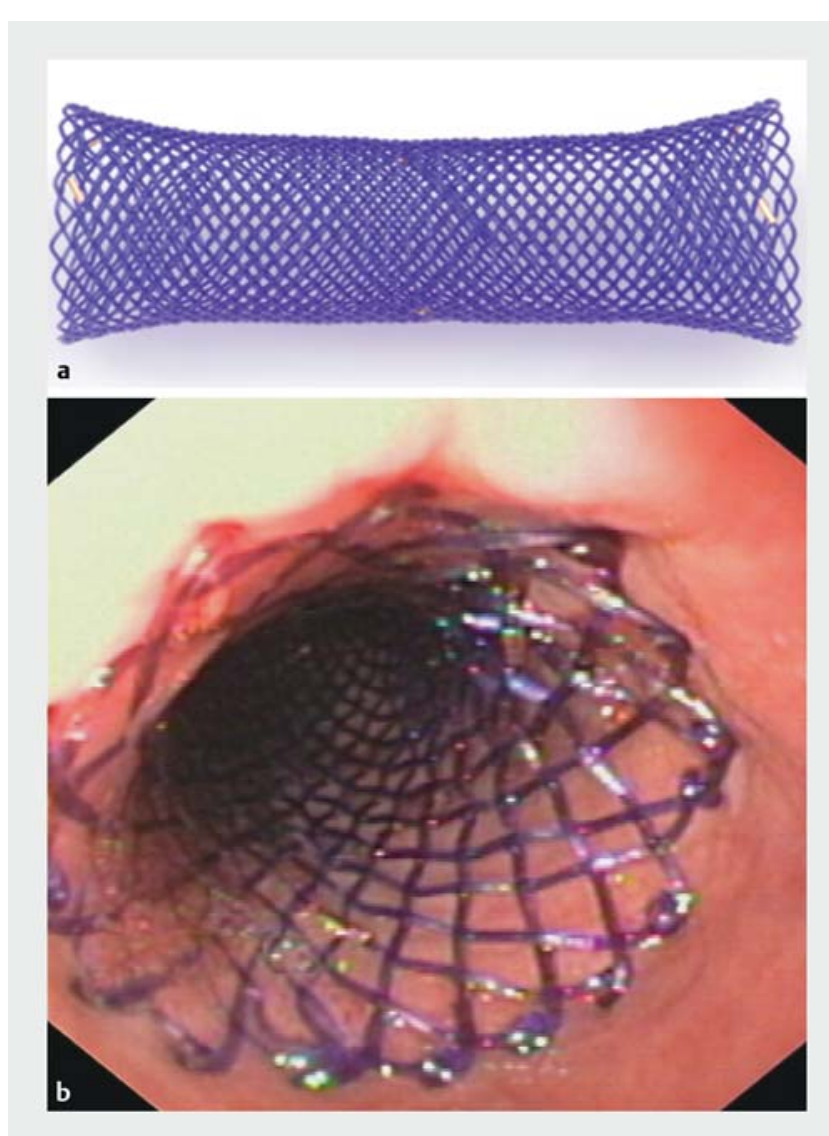

- Fig. 1 Biodegradable stent. a The SX-ELLA stent, with radiopaque markers, made from biodegradable polydioxanone. Stents are available in multiple lengths $(60,80$, and $100 \mathrm{~mm})$ and diameters $(18,20$, and $23 \mathrm{~mm})$. $\mathbf{b}$ Endoscopic image of the biodegradable stent placed across a benign esophageal stricture.

kak and Bennett scale [22], was performed at the physician's discretion. When recurrent significant dysphagia within 6 months of the initial procedure (defined in the dilation group as the procedure in which the final target diameter was reached) occurred in either group, standard dilation up to $18 \mathrm{~mm}$ was performed. When recurrent significant dysphagia occurred after 6 months, all treatment options were available.

\section{Study end points}

The primary end point was the number of repeat endoscopic dilations for recurrent stricture within 3 months and 6 months after stent placement or dilation to $\geq 16 \mathrm{~mm}$. Recurrent stricture was defined as any apparent stricture in patients presenting with dysphagia for at least solid food.

Secondary outcomes through 12 months included safety, freedom from dilation for recurrent stricture, time to first dilation for recurrent stricture, freedom from endoscopic procedures, time to first endoscopy, dysphagia, quality of life, and level of activity.

Safety was reported as the number of non-serious adverse events and serious adverse events (SAE). Dysphagia was assessed using the Ogilvie [21] and Dakkak - Bennett [22] scales (see - Supplementary Table e1, available online). Time to recurrent significant dysphagia was the number of days from the initial procedure to onset of recurrent dysphagia for at least solid food. Quality of life was assessed using the EuroQol (EQ)-5D$3 \mathrm{~L}$, which includes five questions related to health status ( Supplementary Table e1) and a self-reported visual analog scale (VAS) [23]. Collectively, responses to the five questions comprised the composite score. A patient recorded their level of health on a vertical VAS, where the end points were labeled "best imaginable health state" and "worst imaginable health state." Level of activity was assessed using the World Health Organization (WHO) performance score ( $\downarrow$ Supplementary Table e1). Presence of gold markers (biodegradable stent group only) was assessed by radiography.

\section{Statistical analysis}

The Signorini method [24] was used to calculate sample size, and the Holm-Bonferroni method [25] was used to correct for multiple comparisons with two primary hypotheses (i.e. 3 months and 6 months). A Poisson rate of one dilation per patient in 12 weeks in the biodegradable stent group and a Poisson rate of two dilations per patient in 12 weeks in the dilation group was assumed. Sample size calculations resulted in a total sample size of 60 patients with a power of 0.935 . To compensate for a $10 \%$ loss to follow-up, the study enrolled a total of 66 patients.

Continuous variables are expressed as means $( \pm S D)$ or medians (interquartile range $[\mathrm{IQR}]$ and range). Categorical data are presented with percentages. The $t$ test was used to analyze normally distributed continuous data. The Mann - Whitney $U$ test analyzed nonparametric data. The exact Cochran-Armitage test for trend analyzed baseline Ogilvie scores. The chi-squared test or Fischer's exact test was used for categorical variables. Kaplan-Meier analysis was performed to determine freedom from dilation for recurrent stricture, with the $P$ value calculated using the log-rank test. For dysphagia scores, EQ-5D-3L with the self-reported VAS, and WHO performance scores, means were plotted over time with vertical lines representing the $95 \%$ confidence interval. A linear mixed model regression analysis that included follow-up time (continuous, in months), treatment group, and the interaction between follow-up and treatment group corrected for baseline measurements, was used to determine differences between treatment groups while controlling for time. A $P$ value of $<0.05$ was considered to be statistically significant.

\section{Results}

A total of 32 patients were randomized to biodegradable stent placement (biodegradable stent group), and 34 patients were randomized to standard dilation therapy (dilation group, - Fig.2). All patients received the assigned treatment. Baseline characteristics were similar between the groups ( $\bullet$ Table 2 ). The majority of patients in both groups had anastomotic strictures. Prior to stent placement, 11 patients in the biodegradable stent group had pre-dilation up to $16 \mathrm{~mm}$. All stents were successfully placed at the intended location during the initial procedure. 
- Table 2 Patient and lesion characteristics.

\begin{tabular}{|c|c|c|c|}
\hline & Dilation & Stent & $P$ value \\
\hline Patients/lesions, $\mathrm{n}$ & 34 & 32 & - \\
\hline Age, mean $\pm S D$, years & $62 \pm 12$ & $62 \pm 9$ & 0.91 \\
\hline Males, n (\%) & $26(76.5)$ & $21(65.6)$ & 0.42 \\
\hline $\begin{array}{l}\text { Lesion length, cm } \\
\text { (median (n, Q1-Q3, IQR, Range)) }\end{array}$ & $1(33,0.5-2,1.5,0.2-7)$ & $1(26,1-2,1,0.2-7)$ & 0.77 \\
\hline \multicolumn{4}{|l|}{ Diameter of stricture, n (\%) } \\
\hline - Mild (>9.8 mm) & $9(26.5)$ & $11(34.4)$ & \multirow[t]{2}{*}{0.59} \\
\hline - Narrow (<9.8mm) & $25(73.5)$ & $21(65.6)$ & \\
\hline \multicolumn{4}{|l|}{ Morphology of stricture, n (\%) } \\
\hline - Anastomotic stenosis & $26(76.5)$ & $23(71.9)$ & \multirow[t]{4}{*}{0.43} \\
\hline - Caustic stenosis & $2(5.9)$ & $1(3.1)$ & \\
\hline - Peptic stenosis & $3(8.8)$ & $1(3.1)$ & \\
\hline - Other ${ }^{2}$ & $3(8.8)$ & $2(21.9)$ & \\
\hline \multicolumn{4}{|l|}{ Dysphagia score } \\
\hline $\begin{array}{l}\text { - Dakkak-Bennett } \\
\text { (median (n, Q1-Q3, IQR, Range)) }\end{array}$ & $15(34,10-21,11,0-21)$ & $15(32,10-21,11,3-21)$ & 0.93 \\
\hline \multicolumn{4}{|l|}{ - Ogilvie } \\
\hline-0 & $0(0)$ & $0(0)$ & \multirow[t]{5}{*}{0.61} \\
\hline-1 & $0(0)$ & $0(0)$ & \\
\hline-2 & $27(79.4)$ & $22(68.8)$ & \\
\hline-3 & $6(17.6)$ & $10(31.3)$ & \\
\hline-4 & $1(2.9)$ & $0(0)$ & \\
\hline
\end{tabular}

\section{Primary end point: dilation for recurrent stricture}

At 3 months, the biodegradable stent group underwent significantly fewer therapeutic endoscopic dilations for recurrent stricture compared with the dilation group (median 0 vs. 1; $P<0.001$ ) ( $>$ Fig.3a). By 6 months, there was no difference between the groups (median 1 vs. $1 ; P=0.31$ ) ( $\mathbf{F i g . 3 b}$ ).

\section{Mortality and safety}

The non-serious adverse events and the SAEs are shown in $\triangleright \mathbf{T a}$ ble 3. There was no difference between groups in the number of patients experiencing adverse events $(P=0.42)$. The most common adverse event was recurrent significant dysphagia requiring intervention. In the dilation group, two patients experienced perforations. In the biodegradable stent group, patients experienced stent occlusion ( $n=5)$, tracheoesophageal fistula $(n=2)$, and stent migration $(n=1)$.

Eight patients died during the study; none of the deaths were attributed to the study stent by the study sites. In the dilation group, deaths were due to progression of underlying disease (i. e. previous cancer diagnosis; $n=3$ ). In the biodegradable stent group, deaths were due to progression of underlying disease (i. e. previous cancer diagnosis; $n=3$ ) and to respiratory insufficiency and infection subsequent to tracheoesophageal fistula $(n=2)$. One fistula was identified 95 days after initial stent placement and 7 days after placement of a second, larger, nonstudy, biodegradable stent. The second fistula, which was located in an area previously treated by radiotherapy, was identified 96 days after initial stent placement. Subsequently, the patient had multiple surgical interventions, including trachea repair, thoracotomy, tracheal stent placement, and tracheostomy. Both patients subsequently died from respiratory insufficiency and infection. 


\begin{tabular}{|c|c|c|c|c|}
\hline \multicolumn{5}{|c|}{ Assessed for eligibility ( $n=87$ ) } \\
\hline$\longrightarrow$ & \multicolumn{3}{|c|}{$\begin{array}{l}\text { Excluded from study } \\
\text { - Did not meet inclusion criteria } \\
\text { - Met exclusion criteria } \\
\text { - Declined to participate }\end{array}$} & $\begin{array}{r}(n=21) \\
(n=17) \\
(n=4) \\
(n=0)\end{array}$ \\
\hline \multicolumn{5}{|c|}{ Enrollment $(n=66)$} \\
\hline & $\downarrow$ & \multicolumn{2}{|c|}{ Randomization } & \\
\hline \multicolumn{3}{|c|}{$\begin{array}{l}\text { Biodegradable stent } \\
\text { group }(n=32)\end{array}$} & \multicolumn{2}{|c|}{$\begin{array}{l}\text { Dilation group } \\
(n=34)\end{array}$} \\
\hline & & $12-m$ & ollow-up & \\
\hline \multicolumn{3}{|c|}{$\begin{array}{l}\text { - Lost-to follow-up } \\
(n=0) \\
\text { - Discontinued } \\
\text { intervention }(n=3) \\
\text { - Death }(n=5)\end{array}$} & \multicolumn{2}{|c|}{$\begin{array}{l}\text { - Lost-to follow-up } \\
(n=1) \\
\text { - Discontinued } \\
\text { intervention }(n=1) \\
\text { - Death }(n=3)\end{array}$} \\
\hline
\end{tabular}

Fig. 2 Patient flow diagram. Enrollment by original assignment and follow-up through 12 months are shown.

\section{Secondary outcomes}

The biodegradable stent group had a higher rate of freedom from dilation for recurrent stricture compared with the dilation group at 3 months ( $87.5 \%$ vs. $49.5 \%$ ), which was sustained through 6 months ( $48.4 \%$ vs. $34.1 \%$ ) and continued through 12 months $(40.8 \%$ vs. $27.9 \%$; log-rank $P=0.05)$ ( $\triangleright$ Fig. 4$)$. The median time to first dilation of recurrent stricture for the biodegradable stent group was significantly longer than the dilation group (106 and 41.5 days; $P=0.003$; data not shown).

Some patients underwent procedures other than dilation for recurrent stricture, such as removal of food bolus obstruction or evaluation of retrosternal pain. The biodegradable stent group had a higher rate of freedom from endoscopic procedures compared with the dilation group at 3 months (50.0\% vs. $32.4 \%$ ), although the overall number of endoscopic procedures per patient at 3 months was similar between the groups (median 0.5 vs. $1 ; P=0.21$ ). The differences in freedom from endoscopic procedures between groups decreased through 6 months $(30.1 \%$ vs. $23.5 \%)$ and 12 months $(26.3 \%$ vs. $17.6 \%$; $\log$-rank $P=0.26$ ). The median time to first endoscopy was also similar between the groups ( 44 and 28 days; $P=0.54$ ).

Both groups had significantly improved Ogilvie and Dakkak Bennett dysphagia scores at 3 months, 6 months, and 12 months compared with baseline ( $P<0.001$ for all time points). These improvements did not differ between groups $(P=0.68$ [ Fig. 5a], and $P=0.89$ [ $\triangleright$ Fig. 5b]).

Through 12 months, the groups were similar for the EQ-5D composite score $(P=0.57)$ ( $\triangleright$ Fig. 6a). However, patients in the biodegradable stent group reported a significantly better quality of life through 12 months than patients in the dilation group based on the EQ-5D VAS $(P=0.01)(\sim$ Fig. $\mathbf{6 b})$. Level of activity,

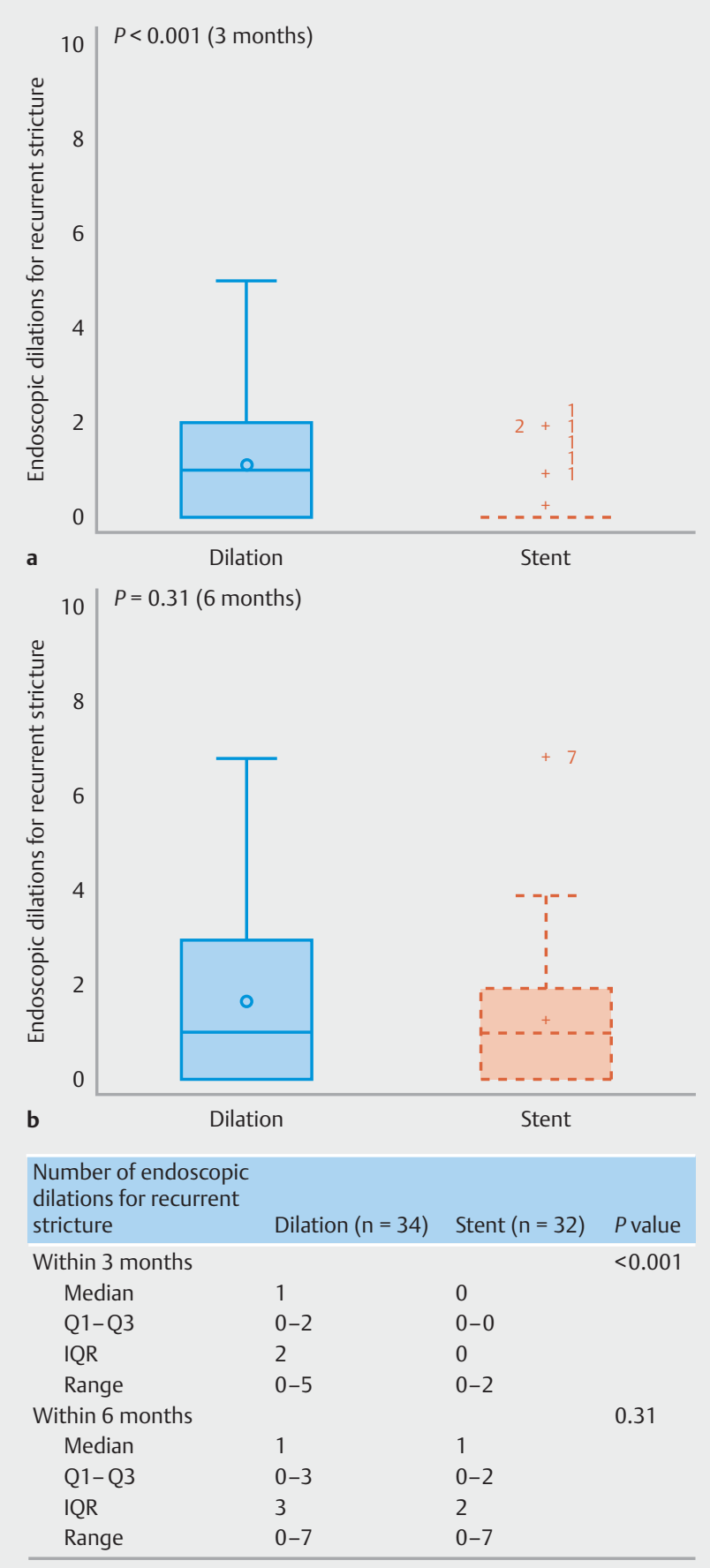

- Fig. 3 Endoscopic dilation for recurrent stricture. a The biodegradable stent group (red bar) underwent significantly fewer endoscopic dilations for recurrent stricture compared with the dilation group (blue bar) at 3 months $(P<0.001)$. $\mathbf{b}$ The number of endoscopic dilations for recurrent stricture between groups was similar by 6 months $(P=0.31)$. Median values are represented by lines; mean values are represented by circles or plus symbols; top whisker is the third quartile (Q3) plus 1.5 times the interquartile range $(\mathrm{IQR}=\mathrm{Q} 3-\mathrm{Q} 1)$; and bottom whisker is $\mathrm{Q} 1$ minus 1.5 times IQR. 
Table 3 Adverse events.

\begin{tabular}{|c|c|c|c|c|}
\hline \multirow[t]{2}{*}{ Event category } & \multicolumn{2}{|c|}{ Non-serious } & \multicolumn{2}{|l|}{ Serious ${ }^{1}$} \\
\hline & Dilation & Stent & Dilation & Stent \\
\hline \multicolumn{5}{|l|}{ Gastrointestinal } \\
\hline - Clinical signs/symptoms² & 11 & 6 & 0 & 5 \\
\hline - Recurrent significant dysphagia requiring intervention & 86 & 71 & 0 & 0 \\
\hline - Occlusion & 0 & 5 & 0 & 0 \\
\hline - Perforation & 0 & 0 & 2 & 0 \\
\hline - Migration & 0 & 0 & 0 & 1 \\
\hline $\begin{array}{l}\text { - Recurrent significant dysphagia requiring intervention requiring } \\
\text { hospitalization }\end{array}$ & 0 & 0 & 2 & 3 \\
\hline - Miscellaneous Gl event ${ }^{3}$ & 10 & 17 & 5 & 2 \\
\hline \multicolumn{5}{|l|}{ Pulmonary } \\
\hline - Tracheoesophageal fistula & 0 & 0 & 0 & 2 \\
\hline - Miscellaneous pulmonary event ${ }^{4}$ & 4 & 3 & 2 & 2 \\
\hline Cardiovascular & 1 & 0 & 1 & 1 \\
\hline Neurologic & 1 & 0 & 1 & 1 \\
\hline Orthopedic & 0 & 2 & 0 & 0 \\
\hline Renal/Urologic & 1 & 1 & 1 & 0 \\
\hline Vascular & 0 & 0 & 0 & 1 \\
\hline Access site/incision & 0 & 0 & 0 & 1 \\
\hline Oncology & 0 & 0 & 4 & 3 \\
\hline Miscellaneous non-Gl event & 11 & 3 & 1 & 1 \\
\hline Total adverse events & 125 & 108 & 19 & 23 \\
\hline \multicolumn{5}{|c|}{$\begin{array}{l}\text { GI, gastrointestinal; SAE, serious adverse event; } \\
{ }^{1} \text { An SAE was defined as an adverse event that led to death, a serious deterioration in the health of the subject resulting in a life-threatening illness or injury or a } \\
\text { permanent impairment of a body structure or body function, required in-patient hospitalization or prolongation of existing hospitalization, resulted in medical or } \\
\text { surgical intervention to prevent permanent impairment to body structure or body function, or led to fetal distress, fetal death, a congenital abnormality, or birth } \\
\text { defect } \\
{ }^{2} \text { Patients may have more than one clinical sign or symptom, which included abdominal pain, nausea, and/or vomiting, as well as retrosternal pain, heartburn, loss of } \\
\text { appetite, regurgitation, and hematemesis } \\
{ }^{3} \text { Serious miscellaneous } \mathrm{Gl} \text { adverse events in the dilation group included esophageal laceration }(n=1) \text {, new symptoms requiring hospitalization }(n=1) \text {, hyperplasia } \\
\text { (metal stent, } n=1) \text {, and follow-up treatment for other condition requiring hospitalization }(n=2) \text {. Serious miscellaneous Gl adverse events in the stent group in- } \\
\text { cluded peritonitis with liver abscess }(n=1) \text { and new symptoms requiring hospitalization }(n=1) \text {. } \\
{ }^{4} \text { Serious miscellaneous pulmonary adverse events in the dilation group included pneumonia }(n=2) \text {. Serious miscellaneous pulmonary adverse events in the stent } \\
\text { group included pneumonia }(n=1) \text { and respiratory insufficiency }(n=1)\end{array}$} \\
\hline
\end{tabular}

measured using the WHO performance score, for patients in the biodegradable stent group was significantly better than the level of activity for patients in the dilation group through 12 months $(P<0.001)$ ( Fig. 6c). Compared with baseline, patients in the biodegradable stent group had significantly improved WHO performance scores at 6 months $(P=0.001)$ and 12 months $(P<0.05)$.

Gold markers were visible in 25 of 29 patients (86.2\%) evaluated in the biodegradable stent group at 3 months. By 6 months, gold markers were visible in 4 of 23 patients (17.4\%). No adverse events related to passing or retention of the gold markers were reported.

\section{Discussion}

The need for frequent repeated endoscopic dilations, which are considered a burden to patients and increase healthcare costs $[5,6]$, is one of the main rationales for seeking an alternative treatment for patients with BES. Initial reports of biodegradable stent placement for BES had disappointing results; however, the more recently available polydioxanone biodegradable stent has resulted in increased placement of biodegradable stents [15-19]. In the current study, patients in the biodegradable stent group had fewer repeat dilations for recurrent stricture within the first 3 months. Furthermore, patients in the biodegradable stent group had a significantly longer time to first di- 


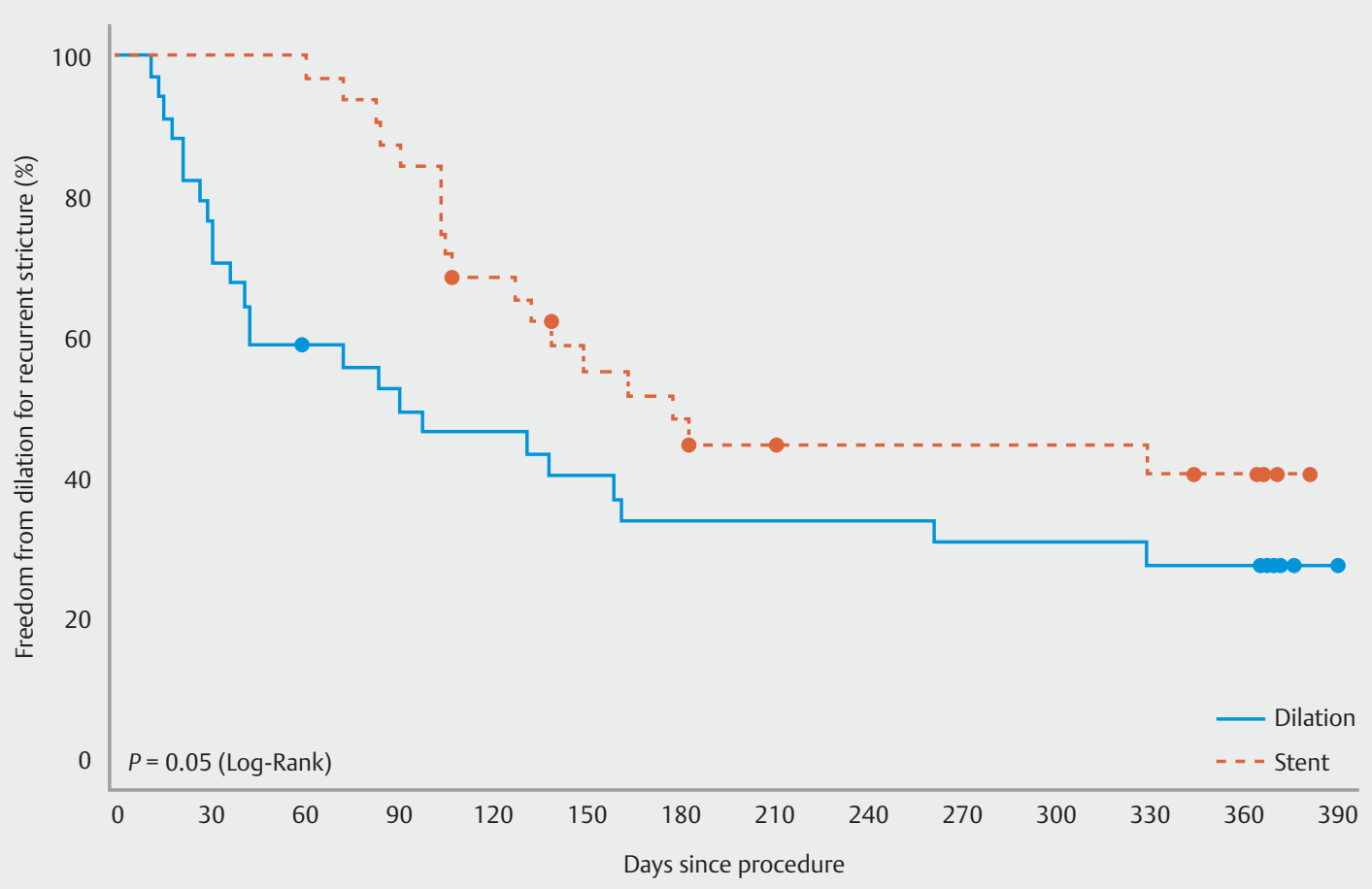

- Fig. 4 First dilation of recurrent stricture. Kaplan - Meier estimates for freedom from dilation for recurrent stricture show that the biodegradable stent group (red dashed line) had a higher rate compared with the dilation group (blue solid line) at both 3 months and 6 months. The groups were similar at 12 months $(P=0.05)$.

lation of recurrent stricture. After the first 3 months, which is approximately the time it takes for the stent to degrade, the number of dilations for recurrent dysphagia increased in the biodegradable stent group, and by 6 months, the total number of dilations was comparable between the groups. The total number of endoscopic procedures was not different after 3 months because a number of patients in the biodegradable stent group presented with retrosternal pain, nausea, and vomiting requiring diagnostic endoscopy. This type of adverse event has previously been reported in patients with biodegradable stents and esophageal self-expanding metal stents (SEMS) [16]. Previous studies have reported events related to retrosternal pain with the use of larger-diameter biodegradable stents (e. g. $25 \mathrm{~mm}$ ) $[16,17]$. Stent stiffness and an inflammatory response in the esophageal mucosa may explain these events $[16,26]$. Taken together, our results suggest that placement of a biodegradable stent may provide a temporary benefit to patients with recurrent BES.

Both groups had significantly improved dysphagia scores, although the study did not correlate the timing of the most recent dilation to dysphagia scores or reinterventions. Through 12 months, the biodegradable stent group reported a significantly better overall health status as measured by the EQ-5D VAS. However, there was no difference between groups on the EQ-5D composite score. The EQ-5D composite score allows the patient to choose from three specific statements in each of the five areas, whereas health state is measured using a VAS, which reflects the overall perception of health status and may be in- fluenced by factors unrelated to the specific measures assessed by the EQ-5D. Within the biodegradable stent group, the WHO performance score significantly improved compared with baseline; however, no difference was seen in the dilation group. Through 12 months, the biodegradable stent group showed a significantly higher level of activity as measured by the WHO performance score compared with the dilation group.

Potential limitations to the current study are that quality of life measures were not assessed immediately prior to or after a reintervention, and the timing of the evaluation in relation to other interventions was not identified. The observed differences in quality of life between groups may be related to the sensitivity of the respective scores within this relatively small population or potential confirmation bias associated with group assignment.

In this study, the number of patients experiencing adverse events was not different between the groups; the most common event reported was recurrent significant dysphagia requiring intervention. In the dilation group, the number of SAEs was considerably higher than previously reported $[11,27]$. The reported rate for laceration and/or perforation following dilation ranges from $0.1 \%$ to $3 \%[11,27]$, compared with $9 \%$ in this study. Notably, one of the two perforations developed after placement of a fully covered SEMS (FCSEMS) for a reintervention at 154 days post-procedure, which highlights that caution should be exercised in this patient population. The second perforation developed during the initial dilation procedure in a pa- 

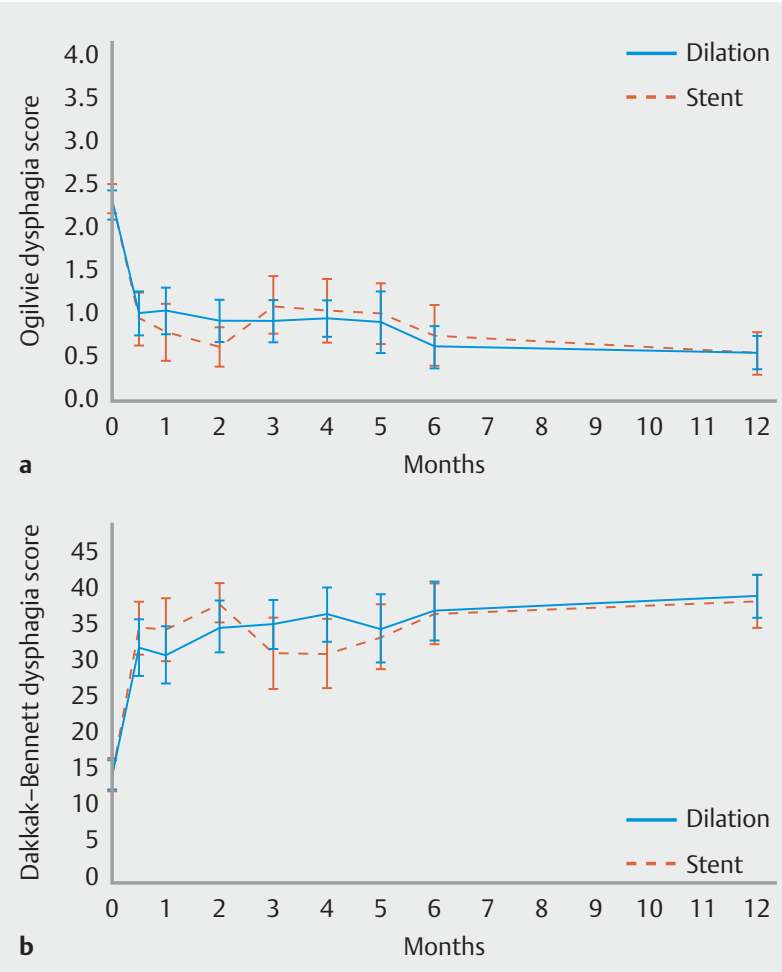

- Fig.5 Mean dysphagia scores over time for the dilation group (blue solid line) and the biodegradable stent group (red dashed line). a Ogilvie dysphagia scores. b Dakkak - Bennett dysphagia scores. Dysphagia scores were similar through 12 months using both the Ogilvie score $(P=0.68)$ and the Dakkak - Bennett $(P=0.89)$ score. Vertical lines represent the $95 \%$ confidence interval for the mean at each time point.

tient with a tortuous and narrow esophageal stricture, which is known to have a higher risk for perforation [11].

Another known risk associated with treating BES is esophagorespiratory fistula formation in patients with esophageal stents. In this study, two patients treated with a biodegradable stent developed a tracheoesophageal fistula approximately 3 months after initial biodegradable stent placement, and later died. In the case where a second, larger, nonstudy, biodegradable stent was placed, the larger stent may have contributed to local tissue damage. In the second case, the fistula was identified in an area where the patient had received radiation treatment for esophageal squamous cell carcinoma; the stent was no longer visible. Radiotherapy in combination with initial radial force from the stent may have contributed to fistula formation. Development of a tracheoesophageal fistula after biodegradable stent placement for a refractory BES has been reported previously $[19,28]$. In a recent study, an esophagobronchial fistula was reported approximately 3 months following placement of a biodegradable stent in a patient with a history of endoscopic submucosal dissection and chemoradiotherapy with repeated endoscopic balloon dilation for refractory BES [19]. The authors suggest caution with use of a biodegradable stent for patients with previous esophageal radiation treatment [19].
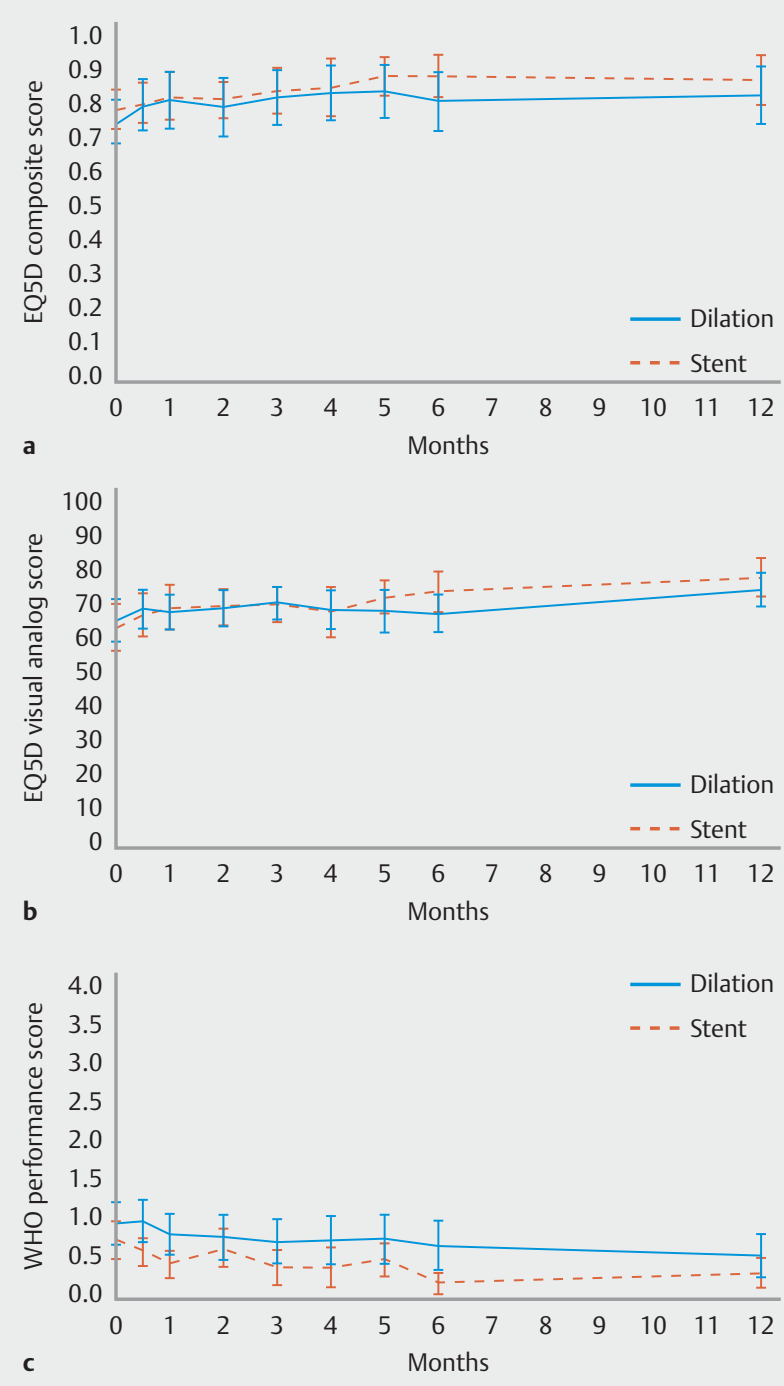

- Fig.6 Quality of life scores over time for the dilation group (blue solid line) and the biodegradable stent group (red dashed line). a Mean EQ-5D composite scores. Through 12 months, the groups were similar $(P=0.57)$. b Mean EQ-5D visual analog scale scores. Patients in the biodegradable stent group reported a significantly better quality of life through 12 months compared with patients in the dilation group $(P=0.01)$. c Mean World Health Organization (WHO) performance scores. The biodegradable stent group had a significantly higher level of activity compared with the dilation group through 12 months $(P<0.001)$. Vertical lines represent the $95 \%$ confidence interval for the mean at each time point.

FCSEMSs are another option for treating BES, but these stents have known complications. Esophagorespiratory fistulas have been reported with the use of SEMS for benign (13.6\%) and malignant $(8.5 \%)$ strictures of the proximal and middle esophagus [29]. Because FCSEMSs are nondegradable stents that require endoscopic removal, biodegradable stents were developed as an alternative. The radial force of the biodegradable stent is typically maintained for up to 8 weeks and decreases over time as the stent degrades $[16,18]$. A flexible stent that has a lower axial force may be preferred; however, no other 
biodegradable stent designs are currently available. Another well-known complication with FCSEMSs is stent migration. In this study, only one partial migration occurred in the biodegradable stent group.

Studies evaluating biodegradable stent placement that include patients with refractory BES have reported a mean clinical success rate of $39 \%$ [20], which is similar to the rate of freedom from endoscopic dilations for recurrent stricture through 12 months in the biodegradable stent group in the current study. Only one randomized study has compared biodegradable stent placement with balloon dilation in patients with BES [26]. However, the study was prematurely closed because of low enrollment; therefore, the study lacked adequate power to determine any statistical differences in dysphagia scores or draw any clinically relevant conclusions. The current study was also challenged by slow patient accrual despite enrollment at eight institutions.

Because the pathogenesis of BES varies, some types of stricture may benefit more from biodegradable stent placement than others, and placement of a biodegradable stent at first presentation with a BES, at least in a subgroup of patients, may have a greater impact. In the current study, most patients presented with anastomotic stricture, suggesting that applicability of the results to BES with other etiology (such as ingestion of caustic substances) may be limited. Furthermore, patients with at least one and a maximum of five previous dilations to $\geq 16 \mathrm{~mm}$ were included in order to ensure that placement of a stent with a minimum diameter of $18 \mathrm{~mm}$ was justified and balanced against the risk of procedure-related complications.

Radiographic visibility of the gold markers served as a surrogate for assessing stent integrity, with the assumption that if the gold markers were not visible, then the biodegradable stent had degraded. By 6 months, gold markers were not visible in the majority of evaluable patients. The timing of stent degradation appears to correspond to the two groups being similar in number of endoscopic dilations for recurrent stricture by 6 months.

There are several limitations to this study. Patients were not blinded to treatment. The type of dilator used by trained physicians was not standardized across the study. Instead, dilation with a balloon or a bougie was performed according to standard institutional practices to reach the target diameter of $\geq 16 \mathrm{~mm}$. In addition, the study did not require a specific algorithm for dilating patients with recurrent stricture after study inclusion. For these patients, dilation was performed as per institutional guidelines. Neither dysphagia scores nor quality of life measures were recorded prior to reintervention.

In conclusion, placement of a biodegradable stent for recurrent $B E S$ is associated with a temporary reduction in the number of repeat dilations and a prolonged time to recurrent dysphagia compared with standard dilation. In general, patients in the biodegradable stent group had improved dysphagia scores and higher level of activity. Although there was no difference between groups in the number of endoscopic dilations for recurrent strictures at 6 months, the biodegradable stent did provide short-term benefits to patients with recurrent BES, the majority of which were anastomotic strictures. Owing to the potential risk of complications, caution should be used when placing a biodegradable stent in patients with previous esophageal radiation treatment. Additional studies are needed to better define the role and the long-term benefit of the biodegradable stent in the treatment of recurrent BES in other subgroups of patients. As the pathogenesis of BES differs, some types of strictures may benefit more from biodegradable stent placement than others.

\section{Acknowledgments}

The authors thank the research staff and the nursing staff for supporting this trial and coordinating data collection. The authors also thank David Wagner of Cook Medical and Scott Snyder, Rachel Bell, and Alicia Altizer of Cook Research Incorporated, a contract research organization, and Cook Group Company, for their assistance in manuscript preparation.

\section{Competing interests}

This study was sponsored by Cook Medical. FP Vleggaar is a consultant for Boston Scientific. A Repici is a consultant for Boston Scientific and has received research fees from Fujifilm Europe, Norgine Europe, and Ferring. PH Deprez is a consultant for Boston Scientific and Olympus. BL Weusten has received consultancy fees from Boston Scientific, and research support from Boston Scientific and C2Therapeutics. R Bisschops has received: consultancy fees from Boston Scientific; speaker fees from Covidien and Norgine; speaker fees and hands-on training sponsorship from Olympus Europe; consultancy fees, speaker fee, and research support from Pentax Europe and Fujifilm; research support from Cook Medical; hands-on training sponsorship from Erbe; and an editorial fee from Thieme Verlag as Co-Editor of Endoscopy. R Haidry has received unrestricted educational grants from Pentax Medical and Cook Endoscopy. KJ Sanborn and EE O'Leary are paid employees of Cook Research Incorporated, a contract research organization and Cook Group Company. JE van Hooft has received research grants from Cook Medical and Abbott, and is a consultant for Boston Scientific and Covidien. PD Siersema is Editor-in-Chief of Endoscopy and has received research grants from Boston Scientific and Cook Medical, and is a consultant for Boston Scientific and EllaCS. 


\section{References}

[1] El-Serag HB. Temporal trends in new and recurrent esophageal strictures in Department of Veterans Affairs. Am J Gastroenterol 2006; 101: $1727-1733$

[2] Raymondi R, Pereira-Lima JC, Valves A et al. Endoscopic dilation of benign esophageal strictures without fluoroscopy: experience of 2750 procedures. Hepatogastroenterology 2008; 55: 1342 - 1348

[3] Ferguson DD. Evaluation and management of benign esophageal strictures. Dis Esophagus 2005; 18: 359-364

[4] Shah JN. Benign refractory esophageal strictures: widening the endoscopist's role. Gastrointest Endosc 2006; 63: 164-167

[5] Dzeletovic I, Fleischer DE, Crowell MD et al. Self dilation as a treatment for resistant benign esophageal strictures: outcome, technique, and quality of life assessment. Dig Dis Sci 2011; 56: 435-440

[6] Martin RC, Woodall C, Duvall R et al. The use of self-expanding silicone stents in esophagectomy strictures: less cost and more efficiency. Ann Thorac Surg 2008; 86: 436-440

[7] Siersema PD, de Wijkerslooth LR. Dilation of refractory benign esophageal strictures. Gastrointest Endosc 2009; 70: 1000-1012

[8] Hordijk ML, van Hooft JE, Hansen BE et al. A randomized comparison of electrocautery incision with Savary bougienage for relief of anastomotic gastroesophageal strictures. Gastrointest Endosc 2009; 70: $849-855$

[9] Pereira-Lima JC, Ramires RP, Zamin I et al. Endoscopic dilation of benign esophageal strictures: report on 1043 procedures. Am J Gastroenterol 1999; 94: 1497-1501

[10] Dua KS, Vleggaar FP, Santharam R et al. Removable self-expanding plastic esophageal stent as a continuous, non-permanent dilator in treating refractory benign esophageal strictures: a prospective twocenter study. Am J Gastroenterol 2008; 103: 2988-2994

[11] de Wijkerslooth LR, Vleggaar FP, Siersema PD. Endoscopic management of difficult or recurrent esophageal strictures. Am J Gastroenterol 2011; 106: 2080 - 2091

[12] van Boeckel PGA, Siersema PD. Refractory esophageal strictures: what to do when dilation fails. Curr Treat Options Gastroenterol 2015; 13: $47-58$

[13] Didden P, Spaander MCW, Bruno MJ et al. Esophageal stents in malignant and benign disorders. Curr Gastroenterol Rep 2013; 15: 319

[14] Seven G, Irani S, Ross AS et al. Partially versus fully covered self-expanding metal stents for benign and malignant esophageal conditions: a single center experience. Surg Endosc 2013; 27: 2185 - 2192

[15] Canena JM, Liberato MJ, Rio-Tinto RA et al. A comparison of the temporary placement of 3 different self-expanding stents for the treat- ment of refractory benign esophageal strictures: a prospective multicentre study. BMC Gastroenterol 2012; 12: 70

[16] Hirdes MM, Siersema PD, van Boeckel PG et al. Single and sequential biodegradable stent placement for refractory benign esophageal strictures: a prospective follow-up study. Endoscopy 2012; 44: 649654

[17] Repici A, Vleggaar FP, Hassan C et al. Efficacy and safety of biodegradable stents for refractory benign esophageal strictures: the BEST (Biodegradable Esophageal Stent) study. Gastrointest Endosc 2010; 72: $927-934$

[18] van Hooft JE, van Berge HenegouwenMI, Rauws EA et al. Endoscopic treatment of benign anastomotic esophagogastric strictures with a biodegradable stent. Gastrointest Endosc 2011; 73: 1043-1047

[19] Yano T, Yoda Y, Nomura S et al. Prospective trial of biodegradable stents for refractory benign esophageal strictures after curative treatment of esophageal cancer. Gastrointest Endosc 2017; 86: 492 499

[20] Lorenzo-Zuniga V, Moreno-de-Vega V, Marin I et al. Biodegradable stents in gastrointestinal endoscopy. World J Gastroenterol 2014; 20 : $2212-2217$

[21] Ogilvie AL, Dronfield MW, Ferguson R et al. Palliative intubation of oesophagogastric neoplasms at fibreoptic endoscopy. Gut 1982; 23 : $1060-1067$

[22] Dakkak M, Bennett JR. A new dysphagia score with objective validation. J Clin Gastroenterol 1992; 14: 99-100

[23] Brooks R. EuroQol: the current state of play. Health Policy 1996; 37 : $53-72$

[24] Signorini DF. Sample size for Poisson regression. Biometrika 1991; 78 : $446-450$

[25] Abdi H. Holm's sequential Bonferroni procedure. In: Salkind N , ed. Encyclopedia of Research Design. Thousand Oaks, California: Sage; 2010: $1-8$

[26] Dhar A, Close H, Viswanath YK et al. Biodegradable stent or balloon dilatation for benign oesophageal stricture: pilot randomised controlled trial. World J Gastroenterol 2014; 20: 18199-18206

[27] Hirdes MMC, van Hooft JE, Koornstra JJ et al. Endoscopic corticosteroid injections do not reduce dysphagia after endoscopic dilation therapy in patients with benign esophagogastric anastomotic strictures. Clin Gastroenterol Hepatol 2013; 11: 795-801

[28] Jung GE, Sauer P, Schaible A. Tracheoesophageal fistula following implantation of a biodegradable stent for a refractory benign esophageal stricture. Endoscopy 2010; 42 Suppl 2: E338-E339

[29] Bick BL, Song LM, Buttar NS et al. Stent-associated esophagorespiratory fistulas: incidence and risk factors. Gastrointest Endosc 2013; 77: $181-189$ 